\title{
Effect of Aerobic Exercise Prescription Combined with Dietary Intervention on MAPK Signal Pathway in Simple Obese Children
}

\author{
Yali Xu ${ }^{1} *$, Peijie Chen ${ }^{2} *$, Ru Wang ${ }^{2}$ \\ ${ }^{I}$ School of Physical Education and Sport, Henan University, Kaifeng, Henan, China \\ ${ }^{2}$ Shanghai University of Sport, Shanghai, China \\ *Corresponding Author.
}

\begin{abstract}
Purpose: Observe the effect of aerobic exercise prescription combined with diet control on MAPK signaling pathways in children with simple obesity, to explore the molecular mechanisms of weight loss and health promotion in children with simple obesity. Methods: 18 male children with simple obesity were given aerobic exercise training for 4 weeks, 6 days a week, twice a day, each exercise for 2 to 2.5 hours. The dietitian formulated the corresponding diet according to the metabolic rate and weight of each subject, and strictly implemented it. And body composition, blood glucose, blood lipids, serum insulin and other indicators were test before and after the experimental. In addition, the mRNA chip was used to randomly detect the gene expression in the MAPK signaling pathway in the peripheral blood of 5 subjects before and after the experiment. Results: (1) after 4 weeks, the body shape indicators decreased significantly $(p<0.05)$; the blood glucose and lipid metabolism indicators decreased significantly $(p<0.05)(2)$ The gene chip test results found that before and after the experiment, a total of 27 genes in the MAPK signaling pathway were changed. These 27 genes were involved in the four pathways of ERK1/2, ERK5, p38 MAPK, and JNK. Conclusion: (1) 4 weeks of aerobic exercise prescription combined with diet control could significantly reduce the body fat rate of simple obese children, and could significantly improve their glucose and lipid metabolism disorder. (2) 4 weeks of aerobic exercise prescription combined with diet control could play a significant role in MAPK signaling pathway of simple obese children.
\end{abstract}

Keywords: Aerobic exercise prescription, gene chip, simple obese children, MAPK

\section{Introduction}

According to the data of China CDC, the obese under the age of 18 in China has reached 120 million ${ }^{[1]}$. Obesity is more harmful to children, which not only affects their growth and development, mental state and intelligence, but also will last until adulthood when the risk of chronic metabolic diseases (diabetes), cardiovascular and cerebrovascular diseases (coronary heart disease, hypertension) and cancer will increase ${ }^{[2]}$.

Many studies have shown that the occurrence and development of obesity is closely related to mitogen-activated protein kinase (MAPK) pathway, and is related to the increase of MAPK activity ${ }^{[3-5]}$.

In view of the high incidence of obesity among children in China and the serious harm and adverse consequences brought by the lowering ages of cardiovascular diseases, it is urgent to prevent and treat childhood obesity. Losing weight by exercising is widely welcomed by the public as a non-invasive and non-drug weight loss method.

Therefore, this study intends to observe the effects of aerobic exercise prescription for simple obese children for 4 weeks and diet control on MAPK signal pathway, so as to provide theoretical basis for the mechanism of losing weight by exercising of obese children and provide experimental basis for the development of exercise prescription.

ISSN: 0010-8189 


\section{Methods}

\subsection{Objects}

We select simple obese children who participated in the summer camp of Shanghai Peak Weight Loss as our research objects. After sports medical examination and exercise load test ${ }^{[6]}, 18$ male simple obese children without sports contraindications were randomly selected. As subjects, their basic situation is shown in Table 1:

Table1 Basic conditions of subjects

\begin{tabular}{ccccc}
\hline Age (year) & Height $(\mathrm{cm})$ & Weight $(\mathrm{kg})$ & BMI $(\mathrm{kg} / \mathrm{m} 2)$ & Body fat\% \\
\hline $13.53 \pm 2.17$ & $163.53 \pm 11.48$ & $82.48 \pm 20.01$ & $30.45 \pm 4.52$ & $43.04 \pm 4.77$ \\
\hline
\end{tabular}

For this study, all subjects and their guardians were informed and had signed the letter of consent. Their accommodation, training, dieting and rest were under unified management. During the whole experiment, special personnel were assigned to take care of the subjects' daily life, catering, training, and strict management was carried out. The experiment lasted for 4 weeks.

\subsection{Exercise tolerance test}

Exercise load test is carried out by treadmill running method, as follows:

(1) The ECG of all subjects is tested in a rest state to make sure that they can accept the test.

(2) First, the subjects walk for 2 minutes at a speed of $4 \mathrm{~km} / \mathrm{h}$. If no positive ECG is found, they will walk for another 2 minutes at a speed of $6 \mathrm{~km} / \mathrm{h}$. After reaching the target heart rate (20\% to $40 \%$ of heart rate reserve) or when abnormal ECG occurs, the exercising will be stopped immediately.

\subsection{Formulation of aerobic exercise prescription}

According to the physical condition and exercise load test results of the above subjects, the individualized aerobic exercise prescription is formulated as follows (Table 2):

Table 2 Aerobic Exercise Prescription

\begin{tabular}{|c|c|}
\hline & Aerobic exercise prescription \\
\hline Subject & Simple obese children \\
\hline Method of exercise & Jogging, swimming, aerobics, badminton, basketball and other events \\
\hline Exercise intensity & $\begin{array}{l}\text { Target heart rate }{ }^{[7]}=\text { rest heart rate+heart rate reserve } \times 20 \% \sim 40 \% \\
\text { (heart rate reserve }=\text { highest heart rate-rest heart rate) } \\
\text { Real-time monitoring of exercise intensity by polar table }\end{array}$ \\
\hline $\begin{array}{l}\text { Exercise time } \\
\text { and frequency }\end{array}$ & $\begin{array}{l}\text { The duration of each exercise is } 2 \text { to } 2.5 \text { hours. After every } 30 \text { minutes of } \\
\text { exercise, rest for } 5 \text { minutes. Take the exercise twice a day, } 6 \text { days a week }\end{array}$ \\
\hline Diet control & All diets are arranged uniformly. See 2.4 for details \\
\hline Attention & $\begin{array}{l}\text { Full-time coaches guide specific sports, and professional medical personnel } \\
\text { supervise the whole process to ensure safety }\end{array}$ \\
\hline
\end{tabular}

2.4 Diet arrangement for the whole process of weight loss

ISSN: 0010-8189

(C) CONVERTER 2021 
According to the metabolic rate and weight of each subject, the corresponding diet was formulated and weighed by nutritionists. It is strictly prohibited to ingest all kinds of extra heat during the experiment. The total daily energy intake of participants is calculated according to the equation: personal standard weight $\times$ heat energy supply per unit weight of extremely light manual labor $(20$ to $25 \mathrm{kal} / \mathrm{kg})$. Wherein, standard weight $=$ height $(\mathrm{cm})-105$. The energy supply ratio of carbohydrate, fat and protein is about $60 \%, 25 \%$ and $15 \%$.

\subsection{Index detection and method}

The following indexes were tested before and after the experiment. We tested the body shape indicators and the blood glucose and lipid metabolism indicators, see Table 3 for details

\subsection{MAPK signal path detection}

The mRNA chip was used to randomly detect the gene expression of MAPK signal pathway in the peripheral blood of 5 subjects before and after the experiment. RT-PCR method was used to verify the accuracy of gene chip detection results.

\subsection{Statistic method}

Using EXCEL software, paired T test method was adopted to analyze the data before and after the experiment. All the data were expressed by mean \pm standard deviation. $\mathrm{P}<0.05$ indicated significant difference, and $\mathrm{P}<0.01$ suggested extremely significant difference.

\section{Results and analysis}

\subsection{Results}

After four weeks' comprehensive intervention with aerobic exercise prescription and diet control, all of the body shape indicators of the subjects decreased significantly $(\mathrm{P}<0.05)$. Moreover, their serum fasting insulin, blood lipid, blood glucose and insulin resistance index were significantly lower than those before the experiment $(\mathrm{p}<0.05)$. (Table 3)

Table 3 Comparison of body shape and function indexes before and after intervention of aerobic exercise prescription

\begin{tabular}{|l|c|c|}
\hline \multicolumn{1}{|c|}{ Indexes } & Pre-test & Pos-test \\
\hline weight $(\mathrm{kg})$ & $82.48 \pm 20.01$ & $74.59 \pm 18.21^{* *}$ \\
\hline BMI $\left(\mathrm{kg} / \mathrm{m}^{2}\right)$ & $30.45 \pm 4.52$ & $27.29 \pm 3.92^{* *}$ \\
\hline Body fat rate $(\%)$ & $43.04 \pm 4.77$ & $40.28 \pm 4.94^{* *}$ \\
\hline chest circumference $(\mathrm{cm})$ & $99.80 \pm 10.49$ & $93.23 \pm 9.96^{* *}$ \\
\hline waist circumference $(\mathrm{cm})$ & $99.09 \pm 11.04$ & $91.46 \pm 10.58^{* *}$ \\
\hline hip circumference $(\mathrm{cm})$ & $103.97 \pm 10.24$ & $98.21 \pm 10.23^{* *}$ \\
\hline waist/ hip ratio & $0.95 \pm 0.05$ & $0.93 \pm 0.05^{* *}$ \\
\hline FBG $(\mathrm{mmol} / \mathrm{L})$ & $82.48 \pm 20.01$ & $74.59 \pm 18.21^{* *}$ \\
\hline FIN $(\mathrm{mmol} / \mathrm{L})$ & $127.45 \pm 50.93$ & $53.01 \pm 41.57 * *$ \\
\hline HOMA2-IR & $2.23 \pm 0.93$ & $0.94 \pm 0.74^{* *}$ \\
\hline TG $(\mathrm{mmol} / \mathrm{L})$ & $1.78 \pm 0.78$ & $0.79 \pm 0.24 * *$ \\
\hline TC $(\mathrm{mmol} / \mathrm{L})$ & $4.87 \pm 0.69$ & $3.52 \pm 0.56^{* *}$ \\
\hline
\end{tabular}

ISSN: 0010-8189 


\begin{tabular}{|l|c|c|}
\hline $\mathrm{HDL}(\mathrm{mmol} / \mathrm{L})$ & $1.32 \pm 0.23$ & $1.13 \pm 0.24 * *$ \\
\hline $\mathrm{LDL}(\mathrm{mmol} / \mathrm{L})$ & $2.95 \pm 0.45$ & $1.81 \pm 0.35^{* *}$ \\
\hline \multicolumn{2}{|c|}{} & $* *:$ compared with Pre-test, $\mathrm{p}<0.01$ \\
\hline
\end{tabular}

The results of gene chip detection showed that after 4 weeks of aerobic exercise intervention, the expression of 27 genes in MAPK signal pathway changed, which involved ERK1/2, ERK5, p38 MAPK and JNK. These 27 genes are KRAS, AKT3, NRAS, CRKL, TGFBR1, NFKB2, PRKACB, PLA2G2A, PLA2G2E, MAP3K2, MAP3K4, MAP3K8, MAP4K3, MAP4K4, MAP4K5, CACNA1C, RASA1, ZAK, DUSP16, DUSP5, ELK4, NR4A1, PPM1B, TAOK2, RPS6KB1, RIPK1.

\subsection{Analysis and discussion}

As can be seen from the above results, aerobic exercise prescription can significantly improve the body composition, body shape and glucose and lipid metabolism disorder of obese children, and can have a significant impact on MAPK signaling pathway.

Studies have shown that the occurrence and development of obesity is closely related to MAPK signaling pathway and the increase of MAPK activity. Many studies have suggested that MAPK signal transduction pathway can participate in the related process of regulating adipocyte differentiation by influencing ERK, p38 MAPK and JNK.

The results of this study showed that after 4 weeks of aerobic exercise intervention, the expression of 27 MAPK signaling pathway related genes changed, which involved ERK1/2, ERK5, p38 MAPK and JNK. It is found that the activation of ERK1/2 pathway is triggered by RAS-RAF-MEK1/2 cascade, and the activation of ERK1/2 pathway can accelerate the proliferation and differentiation of preadipocytes, while the differentiation of adipocytes in the expansion stage is inhibited if treated with MEK inhibitor ${ }^{[8]}$; furthermore, ERK1/2 pathway is necessary for transcription of C-EBP $\alpha / \beta / \delta$ and PPAR $\gamma$ genes, which are key factors of adipocyte differentiation. Bost $\mathrm{F}$ et al. ${ }^{[9]}$ found that the amount of fat cells in ERK1 knockout mice was significantly less than that in non-knockout mice. Moreover, after feeding high-fat diet to ERK1 knockout mice, their postprandial metabolic rate was significantly higher than that of non-knockout mice, and they were less likely to suffer from insulin resistance and obesity.

In addition, the prescription intervention of aerobic exercise in this study can also down-regulate RAS (KRAS, NRAS) and RAS protein activator (RASA1) genes, and RAS is the switch to activate this pathway ${ }^{[10]}$. therefore, it can be known that the intervention of 4 weeks of aerobic exercise can directly inhibit the activation of MEK1/2 pathway, and cause decrease of activity of MEK1/2 pathway through down-regulating other related genes such as NFKB2, CACNA1C, PRKACB, RPS6KB1. On the one hand, it inhibited the differentiation of adipocytes. On the other hand, it activated the apoptosis of mature adipocytes. In this way, it resulted in a significant decrease in the body weight and body fat rate of obese adolescents.

In the ERK5 pathway, the genes involved in changes include MAP4K3, MAP4K4, MAP3K2 and MAP3K8, all of which are down-regulated. The research shows that ERK5 pathway can be activated under the stimulation of serum, epidermal growth factor, reactive oxygen species. According to the conservative three-stage enzymatic cascade reaction, namely $\mathrm{AP} 4 \mathrm{~K} \rightarrow \mathrm{MAP} 3 \mathrm{~K} \rightarrow \mathrm{MAP} 2 \mathrm{~K} \rightarrow \mathrm{MAPK}$, cell proliferation and differentiation was ultimately caused. According to the results of this study, it is speculated that down-regulation of MAP4K3, MAP4K4, MAP3K2 and MAP3K8 genes could weaken the three-stage enzymatic cascade reaction of ERK5 pathway, thus reducing weight and fat by inhibiting the proliferation and differentiation of fat cells of the obese.

In p38 MAPK and JNK pathway, the main genes involved in changes are RAS (KRAS, NRAS), TGFBR, MAP3K4, and MAP4K5, all of which are down-regulated. JNK and p38 MAPK pathways, also known as pressure-activated kinases, can be activated by serum, cytotoxic drugs, radiation, heat stress, reactive oxygen

ISSN: 0010-8189 
species, lipopolysaccharide and other stressors. They are related to cell proliferation and differentiation ${ }^{[11]}$. Aouadi et al. ${ }^{[12]}$ found that p38 MAPK can positively regulate adipogenic differentiation of human preadipocytes, while inhibiting p38 pathway can significantly reduce triglyceride accumulation and expression of related markers. In addition, the experiment of treating obese mice induced by high-fat diet with p38 MAPK specific inhibitors showed that the obesity degree is significantly reduced. The expression of key genes ATF- 2 and PPAR $\gamma 2$ related to adipocyte differentiation is significantly reduced, and adipocyte differentiation is inhibited ${ }^{[13]}$. In addition, a large number of studies have found that JNK pathway is closely related to the development of obesity and apoptosis. Bost $\mathrm{F}^{[14]}$ and other studies have found that compared with the control group, JNK1 gene knock-out (-/-) mice are less likely to become obese after eating high-fat diet. The study of Tuncman et al. ${ }^{[15]}$ shows that the two groups of mice with genotype JNK1 (-/-) and JNK1 (+/-) JNK2(-/-) both have weight loss and increased insulin sensitivity, which are helpful to inhibit obesity. In addition, the experiment of Ahn et al. ${ }^{[16]}$ shows that quercetin can induce apoptosis of mature adipocytes, and the mechanism is that quercetin can inhibit phosphorylation of ERK1/2 and JNK to activate the apoptosis pathway. This experiment showed that ERK1/2 and JNK signaling pathways were closely related with apoptosis of adipocytes. According to the activation circuit diagrams of these two pathways, it can be seen that the down-regulated RAS(KRAS, NRAS), TGFBR, MAP3K4, MAP4K5 and other genes in this study are all at the beginning of the third-order enzymatic cascade reaction, which can directly weaken the activation of p38 MAPK and JNK pathway, and cause the body weight loss and body fat decline by inhibiting adipogenic differentiation of preadipocytes, reducing triglyceride accumulation and activating apoptosis of adipocytes.

\section{Conclusion and suggestion}

4 weeks of aerobic exercise prescription combined with diet control could significantly reduce the body fat rate of simple obese children, and could significantly improve their glucose and lipid metabolism disorder.

4 weeks of aerobic exercise prescription combined with diet control could play a significant role in MAPK signaling pathway of simple obese children, which may lead to the loss of body weight, body fat and insulin resistance by reducing the differentiation of adipocytes and activating the apoptosis of adipocytes.

\section{Reference}

[1] http://www.chinacdc.cn/jlm/mxfcrxjbxx/201302/t20130222_77549.htm

[2] Q. Yang, F. Chen, "Behavioral and psychological factors of obesity," Modern rehabilitation, vol. 5, no. 9, pp. 14-15, 2001.

[3] W. Lu, R.S. Lu, J. Li, et al., "Regulation of MAPK signaling pathway on adipocyte differentiation," Progress in animal medicine, vol. 33, no. 3, pp. 110-114, 2012.

[4] J. Su, H. Su, "Regulatory mechanism of MAPK signaling pathway in adipocyte differentiation," Chemistry of life, vol. 36, no. 2, pp. 252-256, 2016.

[5] B. Chen y, Y.H. Hao, L. Wang, et al., "Molecular mechanism and signal pathway of adipogenic differentiation of adipose derived stem cells," Tissue Engineering Research in China, vol. 21, no. 1, pp. 154-158, 2017.

[6] Y. Guo, P.J. Chen, W.H. Chen, "Effects of 4-week aerobic exercise on body shape, blood lipid and blood insulin in obese children and adolescents," Chinese Journal of sports medicine, vol. 30, no. 5, pp. 427-429, 2011

[7] Y. Guo, W.H. Chen, "Factors influencing obesity and sports weight loss effect," Journal of Shanghai University of Sports, vol. 34, no. 3, pp. 64-66, 2010.

[8] Y.N. Niu, K. Chang, "Research progress of mitogen activated protein kinase signaling pathway in glucose and lipid metabolism," Shanxi Medical Journal, vol. 45, no. 23, pp. 2751-2753, 2016.

[9] F. Bost, M. Aouadi, L. Caron, et al. "The extracellular signal-regulated kinase isoform ERK1 is specifically required for in vitro and in vivo adipogenesis," Diabetes, vol. 54, no. 2, pp. 402-411, 2005. 
[10] H. Yu, X.M. Ma. "Ras mitogen activated protein kinase pathway and tumor targeted therapy," International Journal of oncology, no. 07, pp. 483-485, 2009.

[11] http://www.genome.jp/kegg/pathway/hsa/hsa04010.html

[12] M. Aouadi, J. Jager, K. Laurent, et al. "p38MAP Kinase activity is required for human primary adipocyte differentiation,” FEBS Lett, vol. 581, no. 29, pp.5591-5596, 2007.

[13] T. Maekaw, W. Jin, S. Ishii "The role of ATF-2 family transcription factorsin adipocyte differentiation: antiobesity effects of p38 inhibitors," Mol. Cell. Biol, vol. 30, no. 3, pp.613-625, 2010.

[14] F. Bost, M. Aouadi, L. Caron, et al., "The role of MAPKs in adipocyte differentiation and obesity," Biochimie, vol. 87, no. 1, pp. 51-56, 2005.

[15] G. Tuncman, J. Hirosumi, G. Solinas, et al., "Functional in vivo interactions between JNK1 and JNK2 isoforms in obesity and insulin resistance," Proc. Natl. Acad. Sci. USA, vol. 103, no. 8, pp. 10741-10746, 2006.

[16] J. Ahn, H. Lee, S. Kim, et al., "The anti-obesity effect of quercetin is mediated by the AMPK and MAPK signaling pathways,” Biochem. Biophys. Res. Commun, vol. 373, no. 4, pp. 545-54, 2008. 